\title{
Assessment of Severe Apnoea through Voice Analysis, Automatic Speech, and Speaker Recognition Techniques
}

\author{
Rubén Fernández Pozo, ${ }^{1}$ Jose Luis Blanco Murillo, ${ }^{1}$ Luis Hernández Gómez, ${ }^{1}$ \\ Eduardo López Gonzalo, ${ }^{1}$ José Alcázar Ramírez, ${ }^{2}$ and Doroteo T. Toledano ${ }^{3}$ \\ ${ }^{1}$ Signal, Systems and Radiocommunications Department, Universidad Politécnica de Madrid, Madrid 28040, Spain \\ ${ }^{2}$ Respiratory Department, Hospital Torrecárdenas, Almería 04009, Spain \\ ${ }^{3}$ ATVS Biometric Recognition Group, Universidad Autónoma de Madrid, Madrid 28049, Spain
}

Correspondence should be addressed to Rubén Fernández Pozo, ruben@gaps.ssr.upm.es

Received 1 November 2008; Revised 5 February 2009; Accepted 8 May 2009

Recommended by Tan Lee

This study is part of an ongoing collaborative effort between the medical and the signal processing communities to promote research on applying standard Automatic Speech Recognition (ASR) techniques for the automatic diagnosis of patients with severe obstructive sleep apnoea (OSA). Early detection of severe apnoea cases is important so that patients can receive early treatment. Effective ASR-based detection could dramatically cut medical testing time. Working with a carefully designed speech database of healthy and apnoea subjects, we describe an acoustic search for distinctive apnoea voice characteristics. We also study abnormal nasalization in OSA patients by modelling vowels in nasal and nonnasal phonetic contexts using Gaussian Mixture Model (GMM) pattern recognition on speech spectra. Finally, we present experimental findings regarding the discriminative power of GMMs applied to severe apnoea detection. We have achieved an $81 \%$ correct classification rate, which is very promising and underpins the interest in this line of inquiry.

Copyright ( 2009 Rubén Fernández Pozo et al. This is an open access article distributed under the Creative Commons Attribution License, which permits unrestricted use, distribution, and reproduction in any medium, provided the original work is properly cited.

\section{Introduction}

Obstructive sleep apnoea (OSA) is a highly prevalent disease [1], affecting an estimated $2-4 \%$ of the male population between the ages of 30 and 60 . It is characterized by recurring episodes of sleep-related collapse of the upper airway at the level of the pharynx (AHI > 15, Apnoea Hypopnoea Index, which represents the number of apnoeas and hypoapnoeas per hour of sleep) and it is usually associated with loud snoring and increased daytime sleepiness. OSA is a serious threat to an individual's health if not treated. The condition is a risk factor for hypertension and, possibly, cardiovascular diseases [2], it is usually related to traffic accidents caused by somnolent drivers [1-3], and it can lead to a poor quality of life and impaired work performance. At present, the most effective and widespread treatment for OSA is nasal (Continuous Positive Airway Pressure) CPAP which prevents apnoea episodes by providing a pneumatic splint to the airway. OSA can be diagnosed on the basis of a characteristic history (snoring, daytime sleepiness) and physical examination (increased neck circumference), but a full overnight sleep study is usually needed to confirm the disorder. The procedure is known as conventional Polysomnography, which involves the recording of neuroelectrophisiological and cardiorespiratory variables (ECG). Excellent automatic OSA recognition performance-around $90 \%$ [4] - is attainable with this method based on nocturnal ECG recordings. Nevertheless, this diagnostic procedure is expensive and time consuming, and patients usually have to endure a waiting list of several years before the test is done, since the demand for consultations and diagnostic studies for OSA has recently increased [1]. There is, therefore, a strong need for methods of early diagnosis of apnoea patients in order to reduce these considerable delays.

The pathogenesis of obstructive sleep apnoea has been under investigation for over 25 years, during which a number of factors that contribute to upper airway (UA) collapse during sleep have been identified. Essentially, pharyngeal 
collapse occurs when the normal reduction in pharyngeal dilator muscle tone at the onset of sleep is superimposed on a narrowed and/or highly compliant pharynx. This suggests that OSA may be a heterogeneous disorder, rather than a single disease, involving the interaction of anatomic and neural state-related factors in causing pharyngeal collapse. An excellent review of the anatomic and physiological factors predisposing to UA collapse in adults with OSA can be found in [5]. Furthermore, it is worth noting here that OSA is an anatomic illness, the appearance of which may have been favoured by the evolutionary adaptations in man's upper respiratory tract to facilitate speech, a phenomenon that Jared Diamond calls "The Great Leap Forward" [6]. These anatomic changes include a shortening of the maxillary, ethmoid, palatal and mandibular bones, acute oral cavityskull base angulation, pharyngeal collapse with anterior migration of the foramen magnum, posterior migration of the tongue into the pharynx and descent of the larynx, and shortening of the soft palate with loss of the epiglottic-soft palate lock-up. The adaptations came about, it is believed, partly due to positive selection pressures for bipedalism, binocular vision and the development of voice, speech, and language, but they may also have provided the structural basis for the occurrence of obstructive sleep apnoea.

In our research we investigate the acoustical characteristics of the speech of patients with OSA for the purpose of learning whether severe OSA may be detected using Automatic Speech Recognition techniques (ASR). The automated acoustic analysis of normal and pathological voices as an alternative method of diagnosis is becoming increasingly interesting for researchers in laryngological and speech pathologies in general because of its nonintrusive nature and its potential for providing quantitative data relatively quickly. Most of the approaches found in the literature have focused on parameters based on long-time signal analysis, which require accurate estimation of the fundamental frequency, which is a fairly complex task $[7,8]$. In recent years, some studies have investigated the use of short-time measures for pathological voice detection. Excellent recognition rates have been achieved by modelling short-time speech spectrum information with cepstral coefficients and using statistical pattern classification techniques such as Gaussian Mixture Models (GMMs) $[9,10]$ or discriminative methods such as Support Vector Machines (SVMs) [11]. These techniques based on short-time analyses can provide a characterization of pathologic voices in a direct and noninvasive manner, and so they promise to become a useful support tool for the diagnosis of voice pathologies in general. In our research we are trying to characterize severe apnoea voices in particular.

In this contribution we discuss several ways to apply ASR techniques to the detection of OSA-related traits in specific linguistic contexts. The acoustic properties of voice from speakers suffering obstructive sleep apnoea are not well understood as not much research has been carried out in this area. However, some studies have suggested that certain abnormalities in phonation, articulation, and resonance may be connected to the condition [12]. In order to have a controlled experimental framework to study apnoea voice characterization we collected a speech database [13] designed following linguistic and phonetic criteria we derived from previous research in the field. Our work is focused on continuous speech rather than on sustained vowels, the latter being the standard approach in pathological voice analysis [14]. Therefore, as we are interested in the acoustic analysis of the speech signal in different linguistic and phonetic contexts, our analysis starts with the automatic phonetic segmentation of each sentence using automatic speech recognition based on Hidden Markov Models (HMMs). Together with automatic phonetic segmentation, some basic acoustic processing techniques, mainly related to articulation, phonation, and nasalization, were applied over nonapnoea and apnoea voices to have an initial contrastive study on the acoustic discrimination found in our database. These results provide the proper experimental framework to progress beyond previous research in the field.

After this preliminary acoustic analysis of the discrimination characteristics of our database, we explored the possibilities of using GMM-based automatic speaker recognition techniques [15] to try to observe possible peculiarities in apnoea patients' voices. Successfully detecting traits that prove to be characteristic of the voices of severe apnoea patients by applying such techniques would allow automatic (and rapid) diagnosis of the condition. To our knowledge this study constitutes pioneering research on automatic severe OSA diagnosis using speech processing algorithms on continuous speech. The proposed method is intended as complementary to existing OSA diagnosis methods (e.g., Polysomnography) and clinicians' judgment, as an aid for early detection of these cases. We have observed a marked inadequacy of resources that has led to unacceptable waiting periods. Early severe OSA detection can help increase the efficiency of medical protocols by giving higher priority to more serious cases, thus optimizing both social benefits and medical resources. For instance, patients with severe apnoea have a higher risk of suffering a car accident because of somnolence caused by their condition. Early detection would, therefore, contribute to reducing the risk of suffering a car accident for these patients.

The rest of this document is organized as follows. Section 2 presents the main physiological characteristics of OSA patients and the distinctive acoustic qualities of their voices, as described in the literature. The speech database used in our experimental work, as well as its design criteria, is explained in Section 3. In Section 4 we present a preliminary analysis of the speech signal of the voices in our database, using standard acoustic measurements with the purpose of confirming the occurrence of the characteristic acoustic features identified in previous research. Section 5 explores the advantages that standard automatic speech recognition can bring to diagnosis and monitoring. Next, in Section 6, we describe how we used GMMs to study nasalization in speech, comparing the voices of severe apnoea patients with those in a "healthy" control group. In the same section we also present a test we carried out to assess the accuracy of a GMM-based system we developed to classify speakers (apnoea/nonapnoea). Finally, conclusions and a brief outline of future research are given in Section 7. 


\section{Physiological and Acoustic Characteristics in OSA Speakers}

At present neither the articulatory/physiological peculiarities nor the acoustic characteristics of speech in apnoea speakers are well understood. Most of the more valuable information in this area can be found in Fox and Monoson's work [12], a perceptual study in which skilled judges compared the voices of apnoea patients with those of a control group (referred to as "healthy" subjects). The study showed that, although differences between both groups of speakers were found, acoustic cues for these differences are somewhat contradictory and unclear. What did seem to be clear was that the apnoea group had abnormal resonances that might be due to an altered structure or function of the upper airway. Theoretically, such an anomaly should result not only in respiratory but also in speech dysfunction. Consequently, the occurrence of speech disorder in OSA population should be expected, and it could include anomalies in articulation, phonation, and resonance.

(1) Articulatory Anomalies. Fox and Monoson stated that neuromotor dysfunction could be found in the sleep apnoea population due to a "lack of regulated innervations to the breathing musculature or upper airway muscle hypotonus." This dysfunction is normally related to speech disorders, especially dysarthria. There are several types of dysarthria, resulting in various different acoustic features. All types of dysarthria affect the articulation of consonants and vowels causing the slurring of speech. Another common feature in apnoea patients is hypernasality and problems with respiration.

(2) Phonation Anomalies. These may be due to the heavy snoring of sleep apnoea patients, which can cause inflammation in the upper respiratory system and affect the vocal cords.

(3) Resonance Anomalies. What seems to be clear is that the apnoea group has abnormal resonances that might be due to an altered structure or function of the upper airway causing velopharyngeal dysfunction. This anomaly should, in theory, result in an abnormal vocal quality related to the coupling of the vocal tract with the nasal cavity, and is revealed through two features.

(i) First, speakers with a defective velopharyngeal mechanism can produce speech with inappropriate nasal resonance. The term nasalization can refer to two different phenomena in the context of speech; hyponasality and hypernasality. The former is said to occur when no nasalization is produced when the sound should be nasal. Hypernasality is nasalization during the production of nonnasal (voiced oral) sounds. The interested reader can find an excellent reference in [16]. Fox and Monoson's work on the nasalization characteristics for the sleep apnoea group was not conclusive. What they could conclude was that these resonance abnormalities could be perceived as a form of either hyponasality or hypernasality. Perhaps more importantly, speakers with apnoea may exhibit smaller intraspeaker differences between nonnasal and nasal vowels due to this dysfunction (vowels ordinarily acquire either a nasal or a nonnasal quality depending on the presence or absence of adjacent nasal consonants). Only recently has resonance disorder affecting speech sound quality been associated with vocal tract damping features distinct from airflow in balance between the oral and nasal cavities. The term applied to this speech disorder is "cul-de-sac" resonance, a type of hyponasality that causes the sound to be perceived as if it were resonating in a blind chamber.

(ii) Secondly, due to the pharyngeal anomaly, differences in formant values can be expected, since, for instance, according to [17] the position of the third formant might be related to the size of the velopharyngeal opening (lowering of the velum produces higher third formant frequencies). This is confirmed in Robb et al's work [18], in which vocal tract acoustic resonance was evaluated in a group of OSA males. Statistically significant differences were found in formant frequency and bandwidth values between apnoea and healthy groups. In particular, the results of the formant frequency analysis showed that F1 and F2 values among the OSA group were generally lower than those in the non-OSA groups. The lower formant values were attributed to greater vocal tract length.

These types of anomalies may occur either in isolation or combined. However, none of them was found to be sufficient on its own to allow accurate assessment of the OSA condition. In fact, all three descriptors were necessary to differentiate and predict whether the subject was in the normal group or in the OSA group.

\section{Apnoea Database}

3.1. Speech Corpus. In this section, we describe the apnoea speaker database we designed with the goal of covering all the relevant linguistic/phonetic contexts in which physiological OSA-related peculiarities could have a greater impact. These peculiarities include the articulatory, phonation and resonance anomalies revealed in the previous research review (see Section 2).

As we pointed out in the introduction, the central aim of our study is to apply speech processing techniques to automatically detect OSA-related traits in continuous speech, building on previous perceptual work [12]. Thus, in the present paper we will not be concerned with sustained vowels, even though this has been the most common approach in the literature on pathological voice analysis [14]. This trend no doubt seeks to exploit certain advantages of using sustained vowels, the main one being that their speech signal is more time invariant than that of continuous speech, and therefore it should, in principle, allow a better estimation of the parameters for voice characterization. Another advantage 
for some applications is that certain speaker characteristics such as speaking rate, dialect and intonation do not influence the result. Nevertheless, analysing continuous speech may well afford greater possibilities than working with sustained vowels because certain traits of pathological voice patterns, and in particular those of OSA patients, could then be detected in different sound categories (i.e., nasals, fricatives, etc.) and also in the coarticulation between adjacent sound units. This makes it possible to study the nature of these peculiarities - say, resonance anomalies - in a variety of phonetic contexts, and this is why we have chosen to focus on continuous speech. However, we note that it is not our intention here to compare the performance of continuous speech and sustained vowel approaches.

The speech corpus contains readings of four sentences in Spanish repeated three times by each speaker. Always keeping Fox and Monoson's work in mind, we designed phrases for our speech database that include instances of the following specific phonetic contexts.

(i) In relation to resonance anomalies, we designed sentences that allow intraspeaker variation measurements; that is, measuring differential voice features for each speaker, for instance to compare the degree of vowel nasalization within and without nasal contexts.

(ii) With regard to phonation anomalies, we included continuous voiced sounds to measure irregular phonation patterns related to muscular fatigue in apnoea patients.

(iii) Finally, to look at articulatory anomalies we collected voiced sounds affected by certain preceding phonemes that have their primary locus of articulation near the back of the oral cavity, specifically, velar phonemes such as the Spanish velar approximant "g". This anatomical region has been seen to display physical anomalies in speakers suffering from apnoea. Thus, it is reasonable to suspect that different coarticulatory effects may occur with these phonemes in speakers with and without apnoea. In particular, in our corpus we collected instances of transitions from the Spanish voiced velar plosive /g/ to vowels, in order to analyse the specific impact of articulatory dysfunctions in the pharyngeal region.

All the sentences were designed to exhibit a similar melodic structure, and speakers were asked to read them with a specific rhythmic structure under the supervision of an expert. We followed this controlled rhythmic recording procedure hoping to minimise nonrelevant interspeaker linguistic variability. The sentences used were the following.

(1) Francia, Suiza y Hungría ya hicieron causa común.

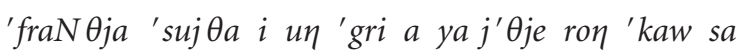
ko'mun

(2) Julián no vio la manga roja que ellos buscan, en ningún almacén.

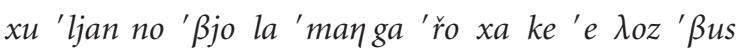
kan en niq 'gun al ma'ken
(3) Juan no puso la taza rota que tanto le gusta en el aljibe.

xwan no'pu so la 'ta $\theta a$ 'ŕo ta ke 'taN to le 'yus ta en el al'xi $\beta e$

(4) Miguel y Manu llamarán entre ocho y nueve y media.

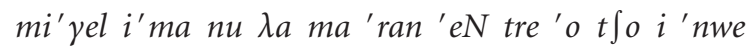
ße i'me ðja

The first phrase was taken from the Albayzin database, a standard phonetically balanced speech database for Spanish [19]. It was chosen because it contains an interesting sequence of successive /a/ and /i/ vowel sounds.

The second and third phrases, both negative, have a similar grammatical and intonation structure. They are potentially useful for contrastive studies of vowels in different linguistic contexts. Some examples of these contrastive pairs arise from comparing a nasal context, "manga roja" (' man ga 'ro $x a$ ), with a neutral context, "taza rota" ('ta $\theta a$ ' ro ta). As we mentioned in the previous section, these contrastive analyses could be very helpful to confirm whether indeed the voices of speakers with apnoea have an altered overall nasal quality and display smaller intraspeaker differences between nonnasal and nasal vowels due to velopharyngeal dysfunction.

The fourth phrase has a single and relatively long melodic group containing mainly voiced sounds. The rationale for this fourth sentence is that apnoea speakers usually show fatigue in the upper airway muscles. Therefore, this sentence may be helpful to discover various anomalies during the sustained generation of voiced sounds. These phonationrelated features of segments of harmonic voice can be characterized following any of a number of conventional approaches that use a set of individual measurements such as the Harmonic to Noise Ratio (HNR) [20], periodicity measures and pitch dynamics (e.g., jitter). The sentence also contains several vowel sounds embedded in nasal contexts that could be used to study phonation and articulation in nasalized vowels. Finally, with regard to the resonance anomalies found in the literature, one of the possible traits of apnoea speakers is dysarthria. Our sentence can be used to analyse dysarthric voices that typically show differences in vowel space with respect to normal speakers [21].

3.2. Data Collection. The database was recorded in the Respiratory Department at Hospital Clinico Universitario of Málaga, Spain. It contains the readings (see Section 3.1) of 80 male subjects; half of them suffer from severe sleep apnoea $(\mathrm{AHI}>30)$, and the other half are either healthy subjects or only have mild OSA $(\mathrm{AHI}<10)$. Subjects in both groups have similar physical characteristics such as age and Body Mass Index (BMI), see Table 1. The speech material for the apnoea group was recorded and collected in two different sessions: one just before being diagnosed and the other after several months under CPAP treatment. This allows studying the evolution of apnoea voice characteristics for a particular patient before and after treatment. 
TABLE 1: Distribution of normal and pathological speakers in the database.

\begin{tabular}{lccccc}
\hline & Number & Mean age & Std. dev. age & Mean BMI & Std. dev. BMI \\
\hline Normal & 40 & 42.2 & 8.8 & 26.2 & 3.9 \\
Apnoea & 40 & 49.5 & 10.8 & 32.8 & 5.4 \\
\hline
\end{tabular}

3.2.1. Speech Collection. Speech was recorded using a sampling frequency of $48 \mathrm{kHz}$ in an acoustically isolated booth. The recording equipment consisted of a standard laptop computer with a conventional sound card equipped with a SP500 Plantronics headset microphone with A/D conversion and digital data exchange through a USB-port.

3.2.2. Image Collection. Additionally, for each subject in the database, two facial images (frontal and lateral views) were collected under controlled illumination conditions and over a flat white background. A conventional digital camera was used to obtain images in 24-bit RGB format, without compression and with $2272 \times 1704$ resolution. We collected these images because simple visual inspections are usually a first step when evaluating patients under clinical suspicion of suffering from OSA. Visual examination of patients includes searching for distinctive features of the facial morphology of OSA such as a short neck, characteristic mandibular distances and alterations, and obesity. To our knowledge, no research has ever been carried out to detect these OSA-related facial features by means of automatic image processing techniques.

\section{Preliminary Acoustic Analysis of the Apnoea Database}

In order to build on the relatively little knowledge available in this area and to evaluate how well our Apnoea Database is suited for the purposes of our research, we first examined some of the standard acoustic features traditionally used for pathological voice characterization, comparing the apnoea patient group and the control group in specific linguistic contexts.

In a related piece of research, Fiz et al. [22] applied spectral analysis on sustained vowels to detect possible apnoea-pathological cases. They used the following acoustic features: maximum frequency of harmonics, mean frequency of harmonics and number of harmonics. They found statistically significant differences between a control group (healthy subjects) and the sleep apnoea group regarding the maximum harmonic frequency for the vowels $/ \mathrm{i} /$ and /e/, it being lower for OSA patients. Another piece of research on the acoustic characterization of sustained vowels uttered by apnoea patients using Linear Predictive Coding (LPC) can be found in [23]. However, these studies do not investigate all of the possible acoustic peculiarities that may be found in the voices of apnoea patients, since focusing solely on sustained vowels precludes the discovery of acoustic effects that occur in continuous speech only in certain linguistic contexts.

Thus the first stage of our contrastive study was a perceptual and visual comparison of frequency representations (mainly spectrographic, pitch, energy and formant analysis) of apnoea and control group speakers. After this we carried out comparative statistical tests on various other acoustic measurements that might reveal distinctive OSA traits. These measurements were computed in specific linguistic contexts using a phonetic segmentation generated with an HMMbased (Hidden Markov Models) automatic speech recognition system. We chose standard acoustic features and tested their discriminative power on normal and apnoea voices. We chose to compare groups using Mann-Whitney $U$ tests because part of the data was not normally distributed.

With this experimental setup, and following up on previous research on the acoustic characteristics of OSA speakers, we searched for articulatory, phonation and resonance anomalies in apnoea-suffering speakers.

(1) Articulatory Anomalies. An interesting conclusion from our initial perceptual contrastive study was that, when comparing the distance between the second (F2) and third formant (F3) for the vowel /i/, clear differences between the apnoea and control groups were found. For apnoea speakers the distance was greater, and this was especially clear in diphthongs with /i/ as the stressed vowel, as in the Spanish word "Suiza" ('suj $\theta a$ ) (See Figure 1). This finding is in agreement with Robb's conclusion that the F2 formant value in the vowels produced by apnoea subjects is lower (and therefore the distance between F3 and F2 is larger) than normal [18].

This finding may be related to the greater length of the vocal tract of OSA patients [18], but also, and perhaps more importantly, to a characteristically abnormal velopharyngeal opening which may cause a shift in the position of the third formant. Indeed, a lowering of the velum (typical in apnoea speakers) is known to produce higher third formant frequencies. We measured the distance between F2 and F3 in the utterances of first test phrase listed above, which contains good examples of stressed i's. We measured absolute distances in spite of the fact that the actual location of the formants is speaker dependent. Nevertheless, we considered that normalization was not necessary because our database contains only male subjects with similar relevant physical characteristics, and the formants should lie roughly in the same regions for all of our speakers. Significant differences were indeed found (Table 2). This fact could support the hypothesis that some form of nasalization is taking place in the case of apnoea speakers.

(2) Phonation Anomalies. In [12] it is reported that the heavy snoring of sleep apnoea patients can cause inflammation and fatigue in the upper airway muscles and may affect the vocal cords. As indicators of these phonation abnormalities we can use various individual measurements such as the Harmonic to Noise Ratio (HNR) and dysperiodicity parameters. 


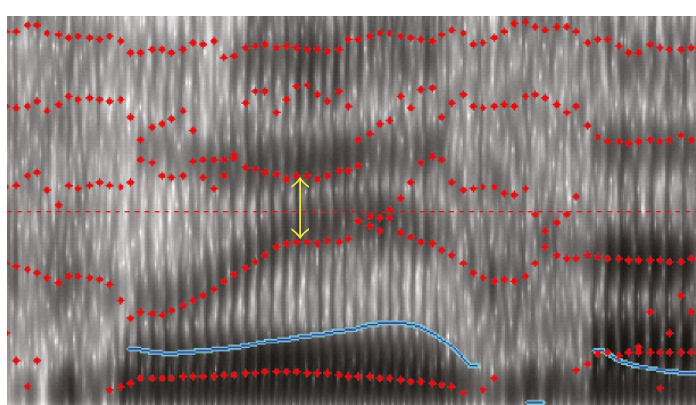

(a)

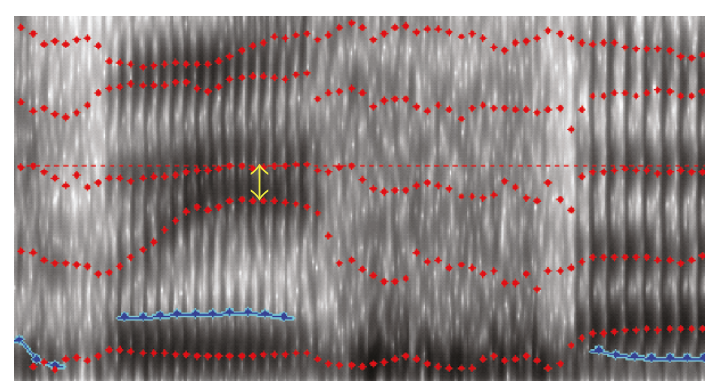

(b)

FIGURE 1: Differences between third and second formant for the vowel "i" in the word "Suiza"('suj $\theta a$ ), (a) for an apnoea speaker and (b) a control group speaker.

TAble 2: Median and $P$-values for articulatory measurements obtained when both groups were compared with the Mann-Whitney $U$ Test.

\begin{tabular}{lccc}
\hline Feature & Group & Median & $\begin{array}{c}P \text {-value } \\
(95 \% \text { conf })\end{array}$ \\
\hline $\begin{array}{l}\text { Dif. third and } \\
\text { second formant }\end{array}$ & $\begin{array}{l}\text { Apnoea } \\
\text { control }\end{array}$ & 614 & $P<.001$ \\
\hline
\end{tabular}

(i) HNR [20] is a measurement of voice pureness. It is based on calculating the ratio of the energy of the harmonics to the noise energy present in the voice (measured in $\mathrm{dB}$ ).

(ii) Dysperiodicity, a common symptom of voice disorders, refers to anomalies in the glottal excitation signal generated by the vibrating vocal folds and the glottal airflow. We estimated vocal dysperiodicities in connected speech following [24].

A normal voice will tend to have a higher HNR and less dysperiodicity (higher signal-to-dysperiodicity ratio) than a "pathological" voice. We computed HNR and signal-todysperiodicity measures for the fourth phrase in the database since it mainly contains voiced sounds and the subjects were asked to read it as a single melodic group. A Mann-Whitney $U$ test revealed significant differences $(P<.05)$ for these measures in the specific linguistics contexts which we stated previously, as we can see in Table 3 . This result suggests that OSA can be linked to certain phonation anomalies, and that the data we collected reveals these phenomena.

(3) Resonance Anomalies. Fox et al. state in [12] that a common resonance feature in apnoea patients is abnormal nasality. The presence and the size of one extra low frequency formant can be considered an indicator of nasalization [25], but no perceptual differences between the groups in the overall nasality level could be found. As discussed in previous sections, this could be due to common perceptual difficulties to classify the voice of apnoea speakers as hyponasal or hypernasal. However, we did find differences in both groups (apnoea and nonapnoea) in how nasalization varied from nasal to nonnasal contexts and vice versa. Interestingly,
TAble 3: Median and $P$-values of phonation measurements obtained when both groups were compared with the Mann-Whitney $U$ Test.

\begin{tabular}{llcc}
\hline Feature & Group & Median & $\begin{array}{c}P \text {-value } \\
(95 \% \text { conf })\end{array}$ \\
\hline HNR & Apnoea & 10.3 & $P=.0110$ \\
Signal-to- & control & 10.6 & \\
dysperiodicity & Apnoea & 30.1 & $P<.001$ \\
\hline
\end{tabular}

we found variation in nasalization to be smaller for OSA speakers. One hypothesis is that the voices of apnoea speakers have a higher overall nasality level caused by velopharyngeal dysfunction, so differences between oral and nasal vowels are smaller than normal because the oral vowels are also nasalized. An explanation for this could be that apnoea speakers have weaker control over the velopharyngeal mechanism, which may cause difficulty in changing nasality levels, whether absolute nasalization level is high or low. These hypotheses are intriguing and we will delve deeper into them later.

\section{Automatic Speech and Speaker Recognition techniques}

When trying to develop a combined model of various features by observing sparse data, statistical modelling is considered to be an adequate solution. Digital processing of speech signals allows performing several parameterizations of the utterances in order to weight up the various dimensions of the feature space, and therefore aim to outline a proper modelling space. Parameters extracted from a given data set, combined with heuristic techniques, will, hopefully, describe a generative model of the group's feature space, which may be compared to others in order to identify common features, analyze existing variability, determine the statistical significance of certain features, or even classify entities. Selecting a convenient parameterization is therefore a relevant task, and one that depends significantly on the specific problem we are dealing with. 
Every sentence in our speech database was processed using short-time analysis with a 20 milliseconds time frame and a 10 milliseconds delay between frames, which gives a $50 \%$ overlap. Each of the windows analyzed will later be presented in the form of a training vector for our statistical models (both HMMs and GMMs). However, before training it is of great importance, as we have already pointed out, to choose an appropriate parameterization for the information. For the task of acoustical space modelling we chose to use 39 standard components: $12 \mathrm{Mel}$ Frecuency Cepstral Coefficients (MFCCs), plus energy, extended with their speed (delta) and acceleration (delta-delta) components. (We acknowledge that an optimized representation-similar to that of Godino et al., for laryngeal pathology detection [9]—could produce better results, but this would require specific adaptation of the recognition techniques to be applied, which falls beyond the goals of the study we present here.) The vectors resulting from this front-end process are placed together in training sets for statistical modelling. This grouping task can be carried out following a variety of criteria depending on the features we are interested in or the phonetic classes that need to be modelled.

As we explained in Section 4, after speech signal parameterization we extract sequences of acoustic features corresponding to specific phonetic and linguistic contextswe believe they may reveal distinctive voice characteristic for OSA speakers. We used well-known speech and speaker recognition techniques to carry out speech phonetic segmentation and apnoea/nonapnoea voice classification.

Since we needed to consider specific acoustical features and phonetic contexts, we first performed a phonetic segmentation of every utterance in the database. This allows combining speech frames from different phonetic contexts for each sound in order to generate a global model, or classifying data by keeping them in separate training sets. For each sentence in the speech database, automatic phonetic segmentation was carried out using the open-source HTK tool [26]. A full set of 24 contextindependent phonetic Hidden Markov Models (HMMs) was trained on a manually phonetically tagged subcorpus of the Albayzin database [18]. As our speech apnoea database includes the transcription of all the utterances, forced segmentation was used to align a phonetic transcription using the 3 -state context-independent HMMs; optional silences between words were allowed to model optional pauses in each sentence. Using automatic forced alignment avoids the need for costly annotation of the data set by hand. It also guarantees good quality segmentation, which is crucial if we are to distinguish phonemes and phonetic contexts.

After phonetic segmentation, statistical pattern recognition can be applied to classify, study or compare apnoea and nonapnoea (control) voices for specific speech segments belonging to different linguistic and phonetic contexts. As cepstral coefficients may follow any statistical distribution on different speech segments, the well-known Gaussian Mixture Model (GMM) approach was chosen to fit a flexible parametric distribution to the statistical distribution of the selected speech segment. Figure 2 summarizes the whole process we have described, showing the direct training of the GMMs from a given database.

In our case we decided to train a universal background GMM model (UBM) from phonetically balanced utterances taken from the Albayzin database [18], and use MAP (Maximum a Posteriori) adaptation to derive the specific GMMs for the different classes to be trained. This technique increases the robustness of the models especially when sparse speech material is available [15]. Only the means were adapted, as is classically done in speaker verification. Figure 3 illustrates the GMM training process.

For the experiments discussed below, both processes, generation of the UBM and MAP adaptation to train the apnoea and the control group GMM models, were developed with the BECARS open source tool [27].

For testing purposes, and in order to increase the number of tests and thus to improve the statistical relevance of our results, the standard leave-one-out testing protocol was used. This protocol consists in discarding one sample speaker from the experimental database to train the classifier with the remaining samples. Then the excluded sample is used as the test data. This scheme is repeated until a sufficient number of tests have been performed.

\section{Apnoea Voice Modelling with GMMs}

In this section we present experimental results that shed light on the potential of using GMMs to discover and model peculiarities in the acoustical signal of apnoea voices, peculiarities which may be related to the perceptually distinguishable traits described in previous research and corroborated in our preceding contrastive study. The main reason for using GMMs over the cepstral domain is related to the great potential this combination of techniques has shown for the modelling of the acoustic space of human speech, for both speech and speaker recognition. For our study we required a good modelling of the anomalies described in Section 2, which we expected to find in OSA patients. Since cepstral coefficients are related with the spectral envelope of speech signals, and therefore with the articulation of sounds, and since GMM training sets can be carefully selected in order to model specific characteristics (e.g., in order to consider resonance anomalies in particular), it seems promising to combine all this information in a fused model. We should expect such a model to be useful for describing the acoustic spaces of both the OSA patient group and the healthy group, and for discriminating between them.

This approach was applied to specific linguistic contexts obtained from our HMM-based automatic phonetic segmentation. In particular, as our apnoea speech database was designed to allow a detailed contrastive analysis of vowels in oral and nasal phonetic contexts, we focus on reporting perceptual differences related to resonance anomalies that could be perceived as either hyponasality or hypernasality. For this purpose, Section 6.1 discusses how GMM techniques can be applied to study these differences in degree of nasalization in different linguistic contexts. After this prospective research, Section 6.2 presents experimental results to test the potential of applying these standard techniques to the automatic 


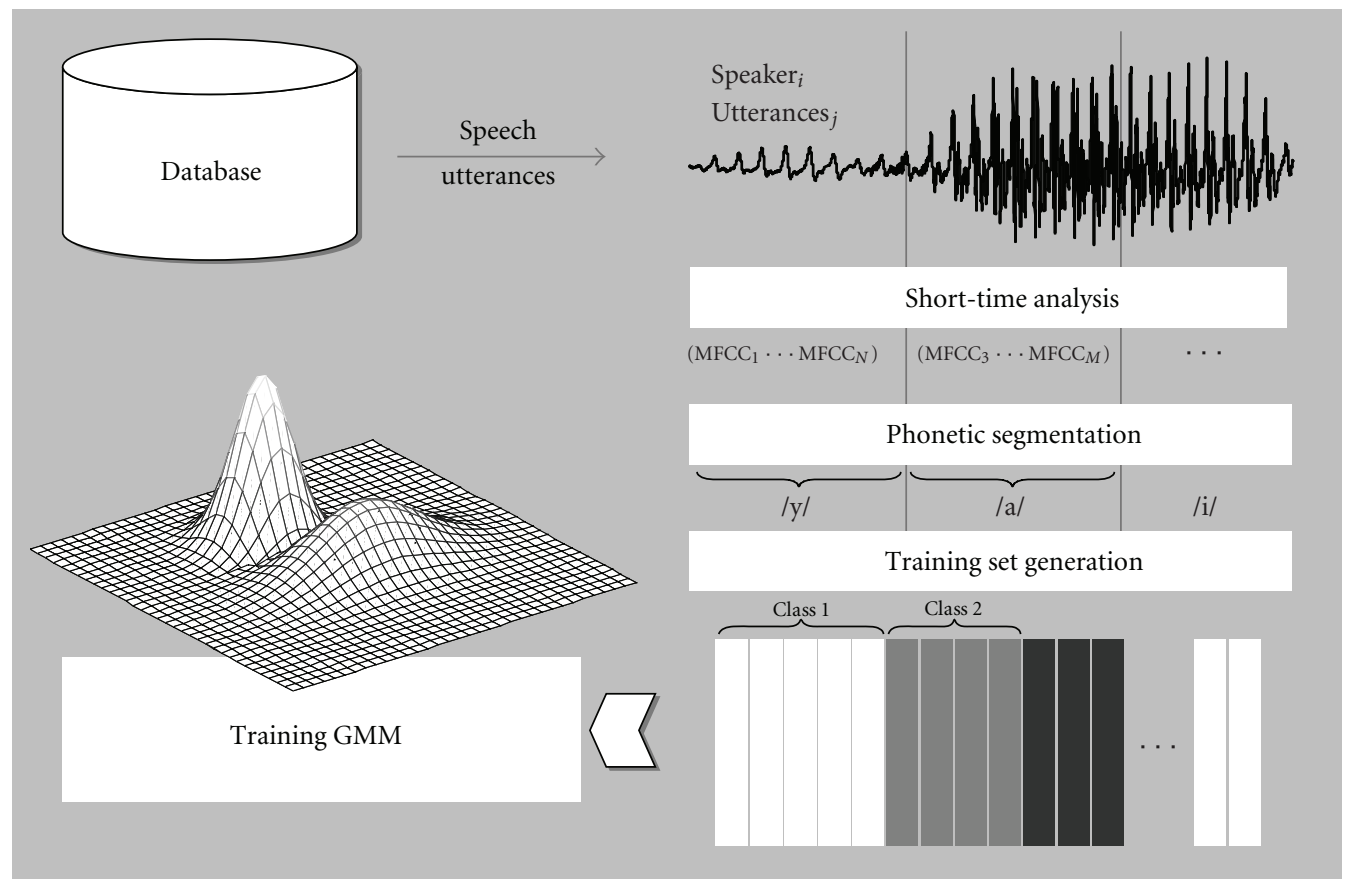

FIgURE 2: Phonetic class GMM model training.

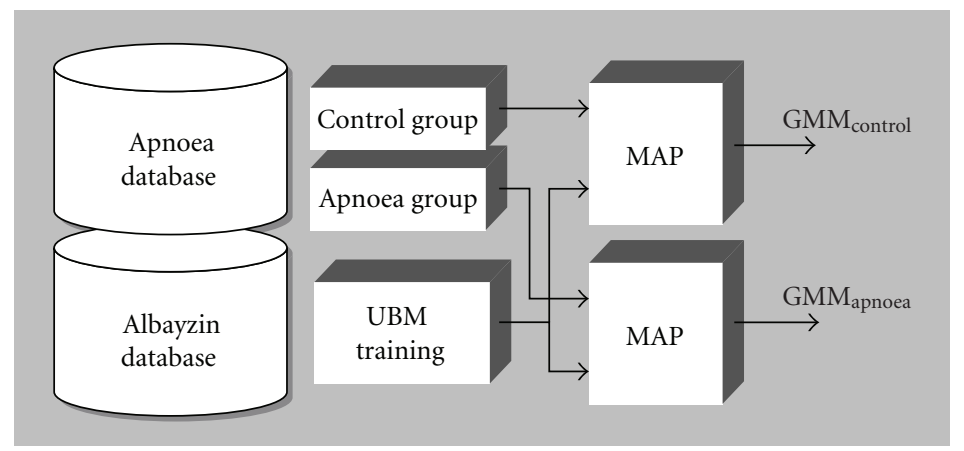

FIGURE 3: Apnoea and control GMM model training.

diagnosis of apnoea, and demonstrate the discriminative power of GMM techniques for severe apnoea assessment.

\subsection{A study of Apnoea Speaker Resonance Anomalies Using} GMMs. To our knowledge, signal processing and pattern recognition techniques have never been used to analyse hyponasal or hypernasal continuous speech from OSA patients. Our aim with the GMM-based experimental setup was to try to model certain resonance anomalies that have already been described for apnoea speakers in preceding research [12] and revealed in our own contrastive acoustic study. Our work focuses mainly on nasality, since distinguishing traits for speakers with apnoea have traditionally been sought in this acoustical aspect.

We therefore used GMM techniques to perform a contrastive analysis to identify differences in degree of nasalization in different linguistic contexts. Two GMMs for each apnoea or healthy speaker were trained using speech with nasalized and nonnasalized vowels. Both speakerdependent nasal and nonnasal GMMs were trained following the approach described in Section 5. MAP adaptation was carried out with a generic vowel UBM trained using Albayzin database [18]. These two nasal/nonnasal GMMs were used to quantify the acoustic differences between nasal and nonnasal contexts for each speaker in both the apnoea and the control groups. The smaller the difference between the nasal and the nonnasal GMMs the more similar the nasalized and the nonnasalized vowels are. Unusually similar nasal and nonnasal vowels for any one speaker reveals the presence of resonance anomalies. We took a fast approximation of the Kullback-Leibler $(K L)$ divergence for Gaussian Mixture Models (Do, 2003) [28] as a measure of distance between nasal and nonnasal GMMs. This distance is commonly used in Automatic Speaker Recognition to define cohorts or groups of speakers producing similar sounds. 
We found that the distance between nasal and nonnasal vowel GMMs was significantly larger for the control group speakers than for the speakers with severe apnoea (a MannWhitney $U$ test revealed significant differences $(P<.05)$ for these distance measures). This interesting result confirms that the margin of acoustic variation for vowels articulated in nasal versus nonnasal phonetic contexts is narrower than normal in speakers with severe apnoea. It also validates the GMM approach as a powerful speech processing and classification technique for research on OSA voice characterization and the detection of OSA speakers.

6.2. Assessment of Severe Apnoea Using GMMs. As we have suggested in the previous section, with the GMM approach we can identify some of the resonance anomalies of apnoea speakers that have already been described in the literature. With our experiment we intended to explore the possibilities that applying GMM-Based Speaker Recognition techniques may open up for the automatic diagnosis of severe apnoea. A speaker verification system is a supervised classification system capable of discriminating between two classes of speech signals (usually "genuine" and "impostor"). For our present purposes the classes are not defined by reference to any particular speaker. Rather, we generated a general severe sleep apnoea class and a control class (speech from healthy subjects) by grouping together all of the training data from speakers of each class and directly applying the appropriate algorithm to fit both Gaussian mixtures onto our data, because what we are interested in is in being able to classify people (as accurately as possible) as either suffering from severe OSA or not. This method is suitable for keeping track of the progress of voice dysfunction in OSA patients, it is easy-to-use, fast, noninvasive and much cheaper than traditional alternatives. While we do not suggest it should replace current OSA diagnosis methods, we believe it can be a great aid for early detection of severe apnoea cases.

Following a similar approach to that of other pathological voice assessment studies [9], GMMs representing the apnoea and control classes were built as follows.

(i) The pathological and control GMMs were trained from the generic UBM relying on MAP adaptation and the standard leave-one-out technique, similarly to how we described above (Section 5).

(ii) During the apnoea/nonapnoea detection phase an input speech signal corresponding to the whole utterance of the speaker to be diagnosed is presented to the system. The parameterised speech is then processed with each apnoea and control GMM generating two likelihood scores. From these two scores an apnoea/control decision is made according to a decision threshold adjusted beforehand as a tradeoff to achieve acceptable rates of both failure to detect apnoea voices (false negative) or falsely classifying healthy cases as apnoea voices (false positive).

Table 4 shows the correct classification rates we obtained when we applied the GMM control/pathological voice classification approach to our speech apnoea database [10]. We see that the overall correct classification rate was $81 \%$.
TABLE 4: Correct classification rate.

\begin{tabular}{lccc}
\hline Correct & Control & Apnoea & Overall \\
classification & group & group & \\
rate in $\%$ & $77.5 \%$ & $85 \%$ & $81 \%$ \\
& $(31 / 40)$ & $(34 / 40)$ & $(65 / 80)$ \\
\hline
\end{tabular}

TABle 5: Contingency table of clinical diagnosis versus automatic classification of patients.

\begin{tabular}{lccc}
\hline & $\begin{array}{c}\text { GMM } \\
\text { classification } \\
\text { severe apnoea }\end{array}$ & $\begin{array}{c}\text { GMM } \\
\text { classification } \\
\text { nonapnoea }\end{array}$ \\
\hline $\begin{array}{l}\text { Diagnosed } \\
\text { severe apnoea } \\
(\text { AHI }>30)\end{array}$ & (A) 40 & $\begin{array}{c}\text { True positive } \\
\text { (TP) } 31\end{array}$ & $\begin{array}{c}\text { False negative } \\
\text { (FN) } 9\end{array}$ \\
$\begin{array}{l}\text { Diagnosed } \\
\text { nonapnoea } \\
(\text { AHI }<10)\end{array}$ & (N) 40 & $\begin{array}{c}\text { False positive } \\
\text { (FP) } 6\end{array}$ & $\begin{array}{c}\text { True negative } \\
\text { (TN) } 34\end{array}$ \\
\hline
\end{tabular}

Table 5 is a contingency table that shows that 31 of the 40 speakers in the database diagnosed with severe apnoea were classified as such by our GMM-based system (true positives), while 9 of them were wrongly classified as nonapnoea speakers (false negatives); and 34 of the 40 speakers diagnosed as not suffering from severe apnoea were classified as such by our GMM-based system (true negatives), while 6 of them were wrongly classified as apnoea speakers (false positives).

Fisher's exact test revealed a significant association $(P<.001)$ between diagnosis and automatic (GMM-based) classification, that is, it is significantly more likely that a diagnosed patient (either with or without apnoea) will be correctly classified by our system than incorrectly classified.

In order to evaluate the performance of the classifier, and so that we may easily compare it with others, we plotted a Detection Error Tradeoff (DET) curve [29], which is a widely employed tool in the domain of speaker verification. On this curve, false positives are plotted against false negatives for different threshold values, giving a uniform treatment to both types of error. On a DET plot, the better the detector, the closer the curve will get to the bottom-left corner. Figure 4 shows the DET curve for our detector. The point marked with a diamond is the equal error rate (EER) point, that is, the point for which the false positive rate equals the false negative rate. We obtained an EER of approximately $20 \%$.

We now evaluate the performance of the classifier using the following criteria.

(i) Sensitivity: ratio of correctly classified apnoeasuffering speakers (true positives) to total number of speakers actually diagnosed with severe apnoea. Therefore, Sensitivity $=\mathrm{TP} /(\mathrm{TP}+\mathrm{FN})$.

(ii) Specificity: ratio of true negatives to total number of speakers diagnosed as not suffering from apnoea. Specificity $=\mathrm{TN} /(\mathrm{TN}+\mathrm{FP})$.

(iii) Positive Predictive Value: ratio of true positives to total number of patients GMM-classified as having a severe apnoea voice. Positive Predictive Value = $\mathrm{TP} /(\mathrm{TP}+\mathrm{FP})$. 


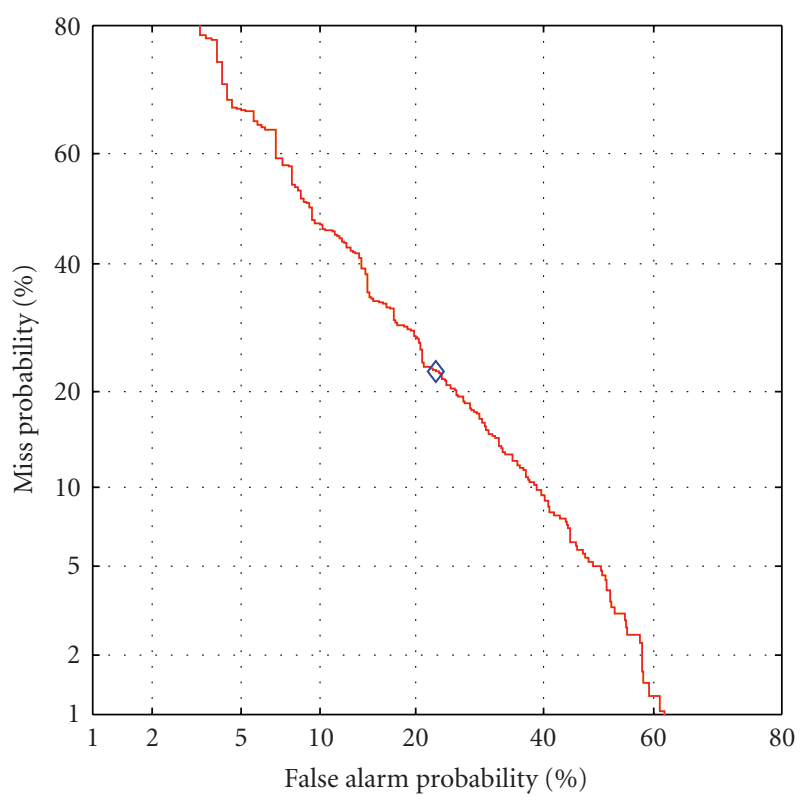

Figure 4: DET plot for our classifier.

Table 6: Sensitivity, specificity, positive and negative predictive value and overall accuracy.

\begin{tabular}{lcccc}
\hline Sensitivity & Specificity & $\begin{array}{c}\text { Positive } \\
\text { predictive } \\
\text { value }\end{array}$ & $\begin{array}{c}\text { Negative } \\
\text { predictive } \\
\text { value }\end{array}$ & $\begin{array}{c}\text { Overall } \\
\text { accuracy }\end{array}$ \\
\hline $77.5 \%$ & $85 \%$ & $83.8 \%$ & $79 \%$ & $81 \%$ \\
$(31 / 40)$ & $(34 / 40)$ & $(31 / 37)$ & $(34 / 43)$ & $(65 / 80)$ \\
\hline
\end{tabular}

(iv) Negative Predictive Value: ratio of true negatives to total number of patients GMM-classified as not having a severe apnoea voice. Negative Predictive Value $=\mathrm{TN} /(\mathrm{TN}+\mathrm{FN})$

(v) Overall Accuracy: ratio of all correctly GMMclassified patients to total number of speakers tested. Overall accuracy $=(\mathrm{TP}+\mathrm{TN}) /(\mathrm{TP}+\mathrm{TN}+\mathrm{FP}+\mathrm{FN})$.

Table 6 shows the values we obtained in our test for these measures of accuracy.

Some comments are in order regarding the correct classification rates obtained. The results are encouraging and they show that distinctive apnoea traits can be identified by a GMM based-approach, even when there is relatively little speech material with which to train the system. Furthermore, such promising results were obtained without choosing any acoustic parameters in particular on which to base the classification. Better results should be expected with a representation and parameterization of audio data that is optimized for apnoea discrimination. Obviously, our experiments need to be validated with a larger test sample. Nevertheless, our results already give us an idea of the discriminative power of this approach to automatic diagnosis of severe apnoea cases.

\section{Conclusions and Future Research}

In this paper we have presented pioneering research in the field of automatic assessment of severe obstructive sleep apnoea. The acoustic properties of the voices of speakers suffering from OSA were studied and an apnoea speech database was designed attempting to cover all the major linguistic contexts in which these physiological OSA features could have a greater impact. For this purpose we analyzed in depth the possibilities of applying standard speechbased recognition systems to the modelling of the peculiar features of the realizations of certain phonemes by apnoea patients. In relation with this issue, we focused on nasality as an important feature in the acoustic characteristics of apnoea speakers. Our state-of-the-art GMM approach has confirmed that there are indeed significant differences between apnoea and control group speakers in terms of relative levels of nasalization between different linguistic contexts. Furthermore, we tested the discriminative power of GMM-based speaker recognition techniques adapted to severe apnoea detection with promising experimental results. A correct classification rate of $81 \%$ shows that GMMbased OSA diagnosis could be useful for the preliminary assessment of apnoea patients and, which suggests it is worthwhile to continue to explore this area.

Regarding future research, our automatic apnoea assessment needs to be validated with a larger sample from a broader spectrum of population. Furthermore, best results can be expected using a representation of the audio data that is optimized for apnoea discrimination. Regarding the decision threshold, an interesting study would be to look at all the possible operating points of the system on a DET curve. It would then be possible to move the system's threshold and fine-tune it to an optimal operating point for medical applications (where, according to common medical criteria, a false negative is a more serious matter than a false positive). Finally, we mention that future research will also be focused on exploiting physiological OSA features in relevant linguistic contexts in order to explore the discriminating power of each feature using linear discriminant classifiers or calibration tools such as the open-source FoCal Toolkit [30]. We aim to apply these findings to improve the performance of the automatic apnoea diagnosis system.

\section{Acknowledgments}

The activities described in this paper were funded by the Spanish Ministry of Science and Technology as part of the TEC2006-13170-C02-02 Project. The authors would like to thank the volunteers at Hospital Clínico Universitario of Málaga, Spain, and to Guillermo Portillo who made the speech and image data collection possible. Also, the authors gratefully acknowledge the helpful comments and discussions of David Díaz Pardo.

\section{References}

[1] F. J. Puertas, G. Pin, J. M. María, and J. Durán, “Documento de consenso Nacional sobre el síndrome de Apneas-hipopneas del sueño (SAHS)," Grupo Español De Sueño (GES), 2005. 
[2] G. Coccagna, A. Pollini, and F. Provini, "Cardiovascular disorders and obstructive sleep apnea syndrome," Clinical and Experimental Hypertension, vol. 28, pp. 217-224, 2006.

[3] P. Lloberes, G. Levy, C. Descals, et al., "Self-reported sleepiness while driving as a risk factor for traffic accidents in patients with obstructive sleep apnoea syndrome and in non-apnoeic snorers," Respiratory Medicine, vol. 94, no. 10, pp. 971-976, 2000.

[4] T. Penzel, J. McNames, P. de Chazal, B. Raymond, A. Murray, and G. Moody, "Systematic comparison of different algorithms for apnoea detection based on electrocardiogram recordings," Medical and Biological Engineering and Computing, vol. 40, no. 4, pp. 402-407, 2002.

[5] C. M. Ryan and T. D. Bradley, "Pathogenesis of obstructive sleep apnea," Journal of Applied Physiology, vol. 99, no. 6, pp. 2440-2450, 2005.

[6] T. M. Davidson, "The great leap forward: the anatomic basis for the acquisition of speech and obstructive sleep apnea," Sleep Medicine, vol. 4, no. 3, pp. 185-194, 2003.

[7] B. Boyanov and S. Hadjitodorov, "Acoustic analysis of pathological voices: a voice analysis system for the screening and laryngeal diseases," IEEE Engineering in Medicine and Biology Magazine, vol. 16, no. 4, pp. 74-82, 1997.

[8] B. Guimarães Aguiar, "Acoustic Analysis and Modelling of Pathological Voices," Microsoft Research, 2007, http:// www.researchchannel.org/prog/displayeventaspx?rID=21533 \&fID $=4834$.

[9] C. Fredouille, G. Pouchoulin, J.-F. Bonastre, M. Azzarello, A. Giovanni, and A. Ghio, "Application of automatic speaker recognition techniques to pathological voice assessment (dysphonia)," in Proceedings of the 9th European Conference on Speech Communication and Technology (Interspeech '05), pp. 149-152, Lisboa, Portugal, September 2005.

[10] J. I. Godino-Llorente, P. Gomes-Vilda, and M. BlancoVelasco, "Dimensionality reduction of a pathological voice quality assessment system based on gaussian mixture models and short-term cepstral parameters," IEEE Transactions on Biomedical Engineering, vol. 53, no. 10, pp. 1943-1953, 2006.

[11] J. I. Godino-Llorente, P. Gómez-Vilda, N. Sáenz-Lechón, M. Blanco-Velasco, F. Cruz-Roldán, and M. A. Ferrer-Ballester, "Support vector machines applied to the detection of voice disorders," in Proceedings of the International Conference on Non-Linear Speech Processing (NOLISP '05), vol. 3817 of Lecture Notes in Computer Science, pp. 219-230, Springer, Barcelona, Spain, April 2005.

[12] A. W. Fox, P. K. Monoson, and C. D. Morgan, "Speech dysfunction of obstructive sleep apnea. A discriminant analysis of its descriptors," Chest, vol. 96, no. 3, pp. 589-595, 1989.

[13] R. Fernandez, L. A. Hernández, E. López, J. Alcázar, G. Portillo, and D. T. Toledano, "Design of a multimodal database for research on automatic detection of severe apnoea cases," in Proceedings of the 6th Language Resources and Evaluation Conference (LREC '08), Marrakech, Morocco, 2008.

[14] V. Parsa and D. G. Jamieson, "Acoustic discrimination of pathological voice: sustained vowels versus continuous speech," Journal of Speech, Language, and Hearing Research, vol. 44, no. 2, pp. 327-339, 2001.

[15] D. A. Reynolds, T. F. Quatieri, and R. B. Dunn, "Speaker verification using adapted Gaussian mixture models," Digital Signal Processing, vol. 10, no. 1, pp. 19-41, 2000.

[16] T. Pruthi, Analysis, vocal-tract modeling and automatic detection of vowel nasalization, Doctor thesis, University of Maryland, Baltimore, Md, USA, 2007.

[17] A. Hidalgo and M. Quilis, Fonética y Fonología Españolas, Tirant Blanch, 2002.
[18] M. P. Robb, J. Yates, and E. J. Morgan, "Vocal tract resonance characteristics of adults with obstructive sleep apnea," Acta Oto-Laryngologica, vol. 117, no. 5, pp. 760-763, 1997.

[19] A. Moreno, D. Poch, A. Bonafonte, et al., "ALBAYZIN speech database: design of the phonetic corpus," in Proceedings of the 3rd European Conference on Speech Communication and Technology (EuroSpeech '93), vol. 1, pp. 175-178, Berlin, Germany, September 1993.

[20] P. Boersma, "Accurate short-term analysis of the fundamental frequency and the harmonics-to-noise ratio of a sampled sound," in Proceedings of the Institute of Phonetic Sciences 17, pp. 97-110, 1993.

[21] G. S. Turner, K. Tjaden, and G. Weismer, "The influence of speaking rate on vowel space and speech intelligibility for individuals with amyotrophic lateral sclerosis," Journal of Speech and Hearing Research, vol. 38, no. 5, pp. 1001-1013, 1995.

[22] J. A. Fiz, J. Morera, J. Abad, et al., "Acoustic analysis of vowel emission in obstructive sleep apnea," Chest, vol. 104, no. 4, pp. 1093-1096, 1993.

[23] A. Obrador, O. Capdevila, M. Monso, et al., "Análisis de la voz en los pacientes con sindrome de apnea-hipopnea en el sueño," in Congreso Nacional de Neumología, 2008.

[24] F. Bettens, F. Grenez, and J. Schoentgen, "Estimation of vocal dysperiodicities in disordered connected speech by means of distant-sample bidirectional linear predictive analysis," Journal of the Acoustical Society of America, vol. 117, no. 1, pp. 328-337, 2005.

[25] J. R. Glass and V. W. Zue, "Detection of nasalized vowels in American english," in Proceedings of IEEE International Conference on Acoustics, Speech, and Signal Processing (ICASSP '85), vol. 10, pp. 1569-1572, Tampa, Fla, USA, April 1985.

[26] S. Young, The HTK Book (for HTK Version 3.2), 2002.

[27] R. Blouet, C. Mokbel, H. Mokbel, E. Sanchez Soto, G. Chollet, and H. Greige, "BECARS: a free software for speaker verification," in Proceedings of the Speaker and Language Recognition Workshop (ODYSSEY '04), pp. 145-148, Toledo, Spain, May-June 2004.

[28] M. N. Do, "Fast approximation of Kullback-Leibler distance for dependence trees and hidden Markov models," IEEE Signal Processing Letters, vol. 10, no. 4, pp. 115-118, 2003.

[29] A. Martin, G. Doddington, T. Kamm, M. Ordowski, and M. Przybocki, "The DET curve in assessment of detection task performance," in Proceedings of the 6th European Conference on Speech Communication and Technology (Eurospeech '97), pp. 1895-1898, Rhodes, Greece, September 1997.

[30] N. Brummer and J. du Preez, "Application-independent evaluation of speaker detection," Computer Speech and Language, vol. 20, no. 2-3, pp. 230-275, 2006. 